\title{
A novel three tier internet of things health monitoring system
}

\author{
Deepika N, M. Anand, F.Jerald \\ Department of Electronics and Communication Engineering, Dr. M. G. R Educational and Research Institute University, \\ India
}

\begin{tabular}{l}
\hline \hline Article Info \\
\hline Article history: \\
Received Dec 19, 2018 \\
Revised Jan 21, 2019 \\
Accepted Mar 10, 2019 \\
\hline
\end{tabular}

Keywords:

Internet of things

Health monitoring system

Cloud computing

\begin{abstract}
Internet of things is a technology where day to day devices, processing, and communication become smarter, intelligent and informative respectively. Health monitoring system is one the most important applications enabled by IOT. IOT health monitoring system comprises of sensors, network and a remote server (cloud). It provides multi-dimensional health monitoring and also suggests treatments. In this paper we have proposed a novel three tier health monitoring system, which stores and process the huge amount of sensor data outside the device using a technology called cloud computing. When the observed clinical sensor value exceeds the threshold clinical value, the system sends alert message to the doctor and caretakers. The history of data and observed data is stored in the cloud for a longer time and it can be viewed by logging into the website.
\end{abstract}

Copyright $\odot 2019$ Institute of Advanced Engineering and Science. All rights reserved.

\section{Corresponding Author:}

Deepika N,

Department of Electronics and Communication Engineering,

Dr. M. G. R Educational and Research Institute University,

Chennai-95, India.

Email: deepikanarayanamoorthy@gmail.com

\section{INTRODUCTION}

Internet of things is a technology that improves the quality of life by allowing all physical objects to communicate with one another and to share useful information with no or less human intervention [1]. It is also defined as the internetworking of devices which uses standard communication protocol to supply services to the users [2].

\section{IOT $=$ Human beings + physical devices + internet}

There is a rapid growth in the number of physical objects connected to the internet which causes a lot of challenges in IOT [3]. Since IOT is at the early stage of development and there is no standard IOT architecture, the communication between Iot things is not secure and it is also subjected to interoperability issue and integration issues [4]. As most of the Iot devices relays on renewable energy and have small sized batteries, the devices must be implemented using low power design. In recent years there is a tremendous increase in the usage of wearable devices for continuous patient health monitoring [5]. IOT wearable devices are attached to the body of patients and physiological parameters are measured continuously [6]. The collected data from the sensors are stored in cloud database for a long period of time. When the medical metrics goes beyond the threshold value, IOT devices sends alert message to the end users.

Cardiovascular disease (CVD) is the major cause of death in the world. Arrhythmia is one of the CVDS which is associated with irregularities in heartbeat. The two major categories of arrhythmias based on single irregular heart beat and a set of irregular heart beat are morphological arrhythmias and rhythmic arrhythmia respectively. The goal of this paper is to implement a three tier health monitoring architecture based of internet of things to detect arrhythmia and implement a web based GUI to view the health status of patients. The health data which are collected from the patient's body will be transmitted to the IoT cloud 
using Wi-Fi because Wi-Fi provides higher data rates and wider coverage areas when compared to Bluetooth or Zigbee. We have deployed HTTP and MQTT servers in the cloud in order to get timely access to the user's health data. To access data in the IOT cloud, a Web based GUI is implemented. This helps the patients and doctors with easy access unlike mobile phones which has different OS.

\section{RELATED WORK}

The use of IOT technology in healthcare has been on increase. Harvard University has developed a project called codeblue for monitoring parameters such as ECG, EEG, EMG, EKG, SpO2 pulse oximeter of an individual [7]. When the health condition of a patient deteriorates, necessary action is taken by doctors. To deliver the health information of patients in a continuous manner, code blue projects has used publish and subscribe architecture [8,9]

University of Virginia has developed a project called alarm-net to measure the health parameters using three tier network architecture [10]. The main aim of this project is to predict critical emergency state of patients using prior health records.

A three tier framework called AID-N has been developed for monitoring the health status of patients [11]. This framework uses light weight algorithm which uses less memory and computational capabilities. Mobihealth is a health monitoring system for ambulant patients. Body sensors are fixed on the patients to measure physiological parameters of patients [12]. The main draw of this system is the lack of security and privacy. Mobicare project has been developed to monitor health of patients and send health information to doctors using fog computing and cloud computing [13].

PAM project has been developed to monitor the mental health condition of patients [14]. PAM framework is used to predict bipolar disorder in advance.

\section{SYSTEM DESIGN AND IMPLEMENTATION}

System architecture as shown in Figure 1.

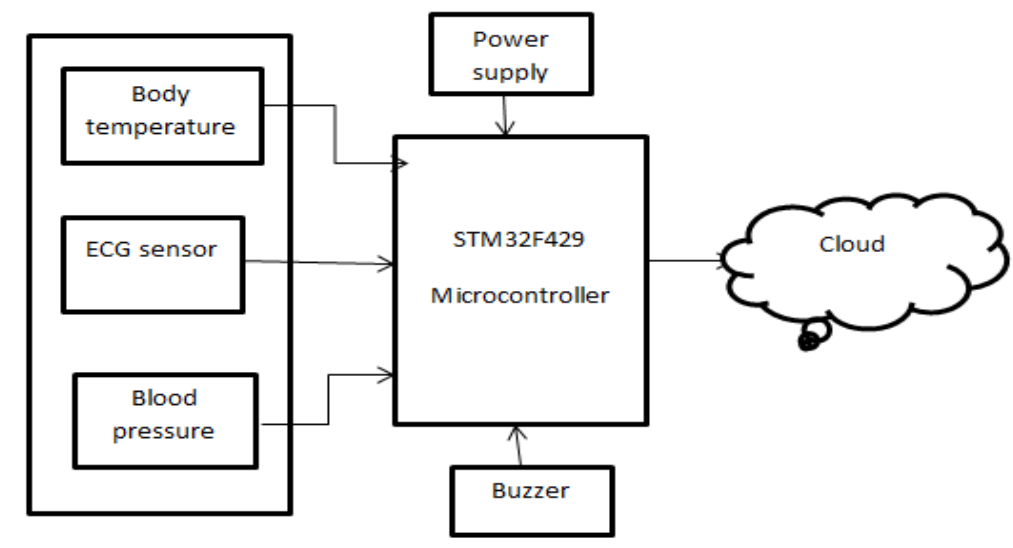

Figure 1. System architecture

\subsection{Sensor Nodes}

A. Temperature sensor

The normal body temperature of human beings depends upon various factors such as recent activities, gender, consumption of liquid and food etc. Normal body temperature of human beings ranges from $97.7 \mathrm{~F}$ to $98.9 \mathrm{~F}$. LM35 temperature sensor is used to measure the body temperature of patients. LM35 is a high precision centigrade temperature sensor which is used to measure temperature. It is a monolithic IC (integrated circuit) that induces an output voltage that is linearly proportional to the Celsius temperature. Temperature measured by LM35 is more accurate than thermistor, and the output voltage generated is higher than thermocouple. Temperature sensors LM35 as shown in Figure 2. 


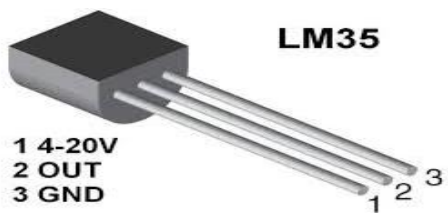

Figure 2. Temperature sensors LM35

\section{B. ECG sensor}

ECG sensor is responsible for the collection of ECG data from the patient's body [13]. AD 8232 ECG sensor and a peripheral circuit is used for collecting ECG data with a higher accuracy rate. The frequency of ECG signals lies between $0.5 \mathrm{~Hz}$ and $100 \mathrm{~Hz}$ [14] and therefore a band pass filter in used in the ECG sensor (AD8232) in order to remove the noise. The collected ECG data has to be processed to boost the signal quality through various techniques such as filtering, amplification, sampling, buffering and packetizing. ECG sensor as shown in Figure 3.

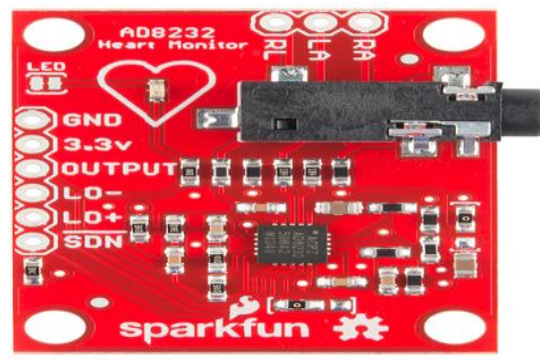

Figure 3. ECG sensor

C. Blood pressure sensor

Blood pressure is an important health parameter which is the pressure of blood in the arteries. There are two types of pressure recorded systolic pressure and diastolic Pressure. To monitor the blood pressure MPX5010GSX sensor is used. Systolic pressure: during heart beat (120mm). Diastolic pressure: during heart relaxation between the beats $(80 \mathrm{~mm})$. ECG signals are collects in a continuous manner; temperature and blood pressure are collected every hour or day.

\subsection{Gateway}

A. STM32F429 microcontroller

Signal processing is implemented in a high performance ARM cortex M-4 microcontroller, with operating at 180 MHZ. STM32F429 and STM32F439 portfolio provides from 512-Kbyte Flash to 2-Mbyte dual-bank Flash, 256-Kbyte SRAM. It supports sleep mode and it is low power consumption MCU which enables long battery life, it is efficient and has easy signal processing capacity [15]. STM32F429 microcontroller as shown in Figure 4.

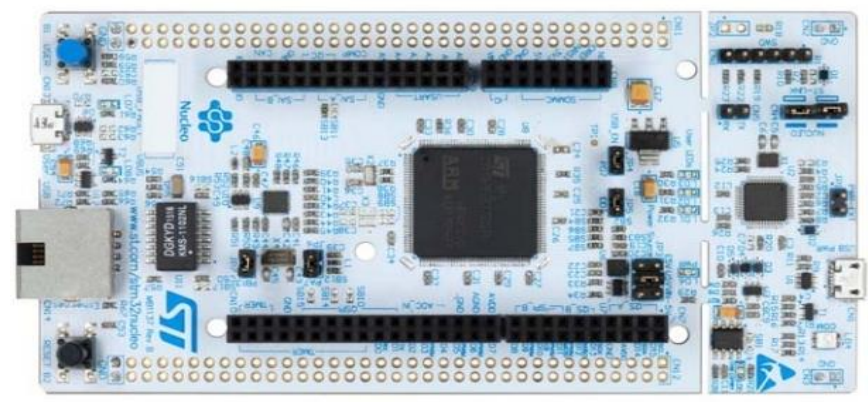

Figure 4. STM32F429 microcontroller 


\section{IMPLEMENTATION OF THREE TIER HEALTH MONITORING SYSTEM}

The proposed health monitoring system consists of a three tier framework which collects, processes, stores and analyses the huge volume of data from the sensor. Tier 1 collects data from the body using wearable devices, tier 2 has a smart gateway which processes the collected data and sends to the cloud for further processing and analysing, tier 3 is iot cloud layer which not only stores data but also predicts abnormalities in the health of patients and sends alert messages to end users.

\subsection{Tier 1: Data Collection}

Sensors are IOT devices which collect physiological data from the human body in a continuous way. The sensor data are sent to the cloud via gateway using Bluetooth. Sensors can be either worn on the body as wearable or implanted in the body. A sensor module consists of four distinct parts:

Power module: it provides energy to the sensor node

Sensor module: it collects data from surrounding and converts the data into electrical signal

Processor module: it controls the sensor and process the collected data

Wireless module: transmits the electrical signal (data) from sensor module to other devices.

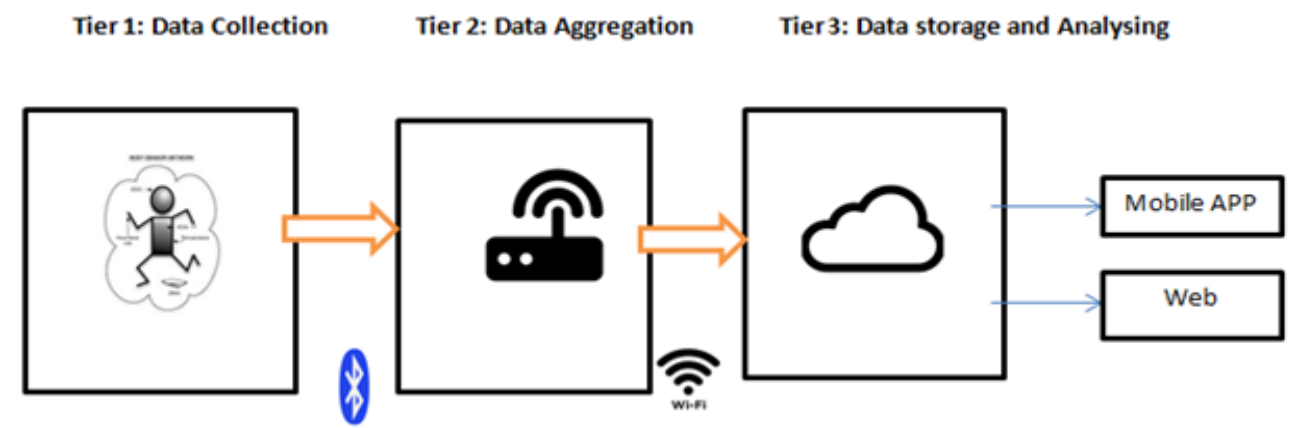

Figure 5. Three tier health monitoring system

\subsection{Tier 2: Data Aggregation}

Sensor data from the IOT device is transmitted to the gateway through Bluetooth and performs data aggregation. Smart gateway acts as a bridge between the sensor layer and IOT cloud. It supports different types of wireless protocols and inter- device communication. It performs protocol conversion, data aggregation, data filtering, data processing and data compressing before sending the data to the IOT cloud. Smart gateway handles a huge amount of data from sensor in a short time and transmits it to the IOT cloud after the following procedures.

Sampling: The signals collected from the sensors are in analog format, which has to be sampled.

Digitalising: Data collected from the sensors are mostly analog signals. Using an analog to digital converter analog signals are converted into digital signals.

Data filtering: As the sensors are very close to the body and in a dynamic environment, noise from the sensor data is removed without important information being lost. The signals were filtered using band pass filter at the frequency of $1-50 \mathrm{HZ}$

Data duplication: When similar readings are transmitted from the sensor, it causes communication overhead and congestion. Therefore data duplication is performed at the smart gateway; If In case the sensor sends same readings.

Packetizing: The sensor data are stored in a buffer and then packetized and sent to the Wi-Fi module. Packetizing is done in order to improve the data transmission efficiency and accuracy.

\subsection{Tier 3: Data Storage and Analysing}

The real time data from the gateway is transmitted to the cloud through Wi-Fi. The huge amount of continuous data is stored in the cloud database and analysed using certain machine learning techniques or data mining approaches. Using this method it is easy to diagnosis heart disease at the early stage. If the analysed clinical data exceed the threshold value alert message will be sent to the doctor and caretakers. By visiting the website or using mobile app, User can log into the iot cloud irrespective of the os platform and obtain the real time sensor data and the history of data. 

protocol

The sensor data from the nodes are transmitted to the webpage using HTPP protocol and MQTT

HTTP: it is a request/ response protocol.

The user sends request to access the webpage, to the HTPP server

In response to the request, the HTPP server sends HTML file, which is converted in to a webpage

MQTT: it is a light weight, publish / subscribe protocol for constrained devices.

The webpage subscribes to topics related to ECG, TEMPERATURE, and BLOOD PRESSURE

Subscribed topic is published to MQTT server and these data is forwarded to the webpage.

Algorithm:

Step 1: IOT devices initialization

Step 2: checks the data from IOT medical sensor

Step 3: if observed value $=$ threshold value

Step 4: stores in cloud database

Step 5: if observed value not equal to threshold value

Step 6: sends alert message to doctor and caretakers.

\section{EXPERIMENTAL RESULT AND DISCUSSION}

\subsection{Experimental Configuration}

Primary features of ECG signals are extracted accurately using a three lead electrode which is placed around the heart and forms a triangle. To diagnose abnormalities we have first set criteria for normal rate. When the ECG pattern, body temperature or blood pressure goes beyond the criteria, warning message will be sent to the user and care takers. We can monitor the variation in body temperature, blood pressure and ECG pattern with graphical representation in mobile phone or website. Table 1 shows the key parameters of the three tier health monitoring system.

Table1. Key Parameters of Three Tier Health Monitoring System

\begin{tabular}{ccc}
\hline & Parameter & Value \\
\hline Sensor & ECG sensor & AD 8283 \\
& Temperature sensor & LM35 \\
& Blood pressure sensor & MPX5010GSX \\
& Power voltage & $3.6 \mathrm{v}$ \\
WiFi module & Wireless transmission protocol & IEEE $802.11 \mathrm{~b} / \mathrm{g} / \mathrm{n}$ \\
server & Database & Redis 3.2 \\
Patient details & Gender & Female \\
& Age & 27 \\
& Height & $172 \mathrm{~cm}$ \\
& Weight & $66 \mathrm{~kg}$ \\
\hline
\end{tabular}

Standard ECG signal: Five types of waves in ECG signals are P wave, T wave, Q wave, R wave, S wave. Various heart diseases are diagnosed using the interval of these five waves.

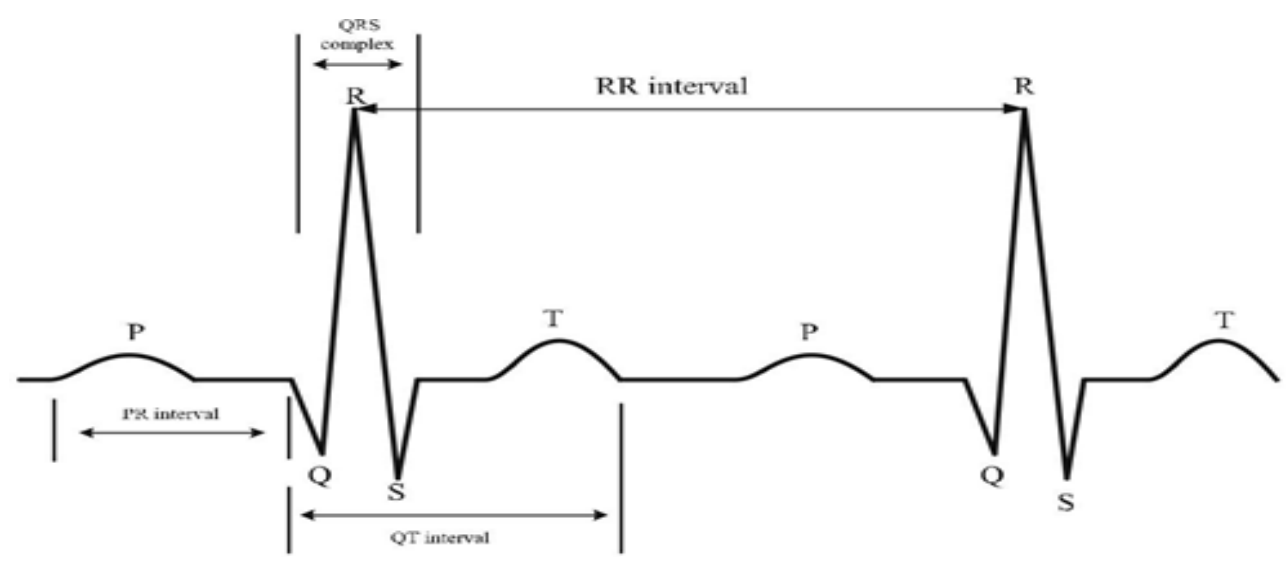

Figure 6. Standard ECG signals 
RR interval: $R$ wave - used to find the period of ECG signal. RR -Time interval between the two adjacent $R$ waves If $R R$ interval is irregular it denotes heart disease like arrhythmia

PR interval: PR - time between the start of $p$ wave and the start of QRS. Time taken by the impulse to reach the ventricles from sinus node

QT interval: QT- time between the beginning and end of Q wave and T wave respectively It is related to ventricular repolarization and ventricular repolarization. It QT interval increases there is a chance of cardiac death.

QRS interval: It consists of three crucial waves Q waves, $\mathrm{R}$ waves, $\mathrm{S}$ waves and it is related with ventricular depolarization

\subsection{Result and Analysis}

Figure 7 shows that the patient's health data can be accessed from the database hosted in the server. The data in the web application is represented as time series as the data is measuring in specific time interval.

\section{Real Time Patient Health Status Monitoring}
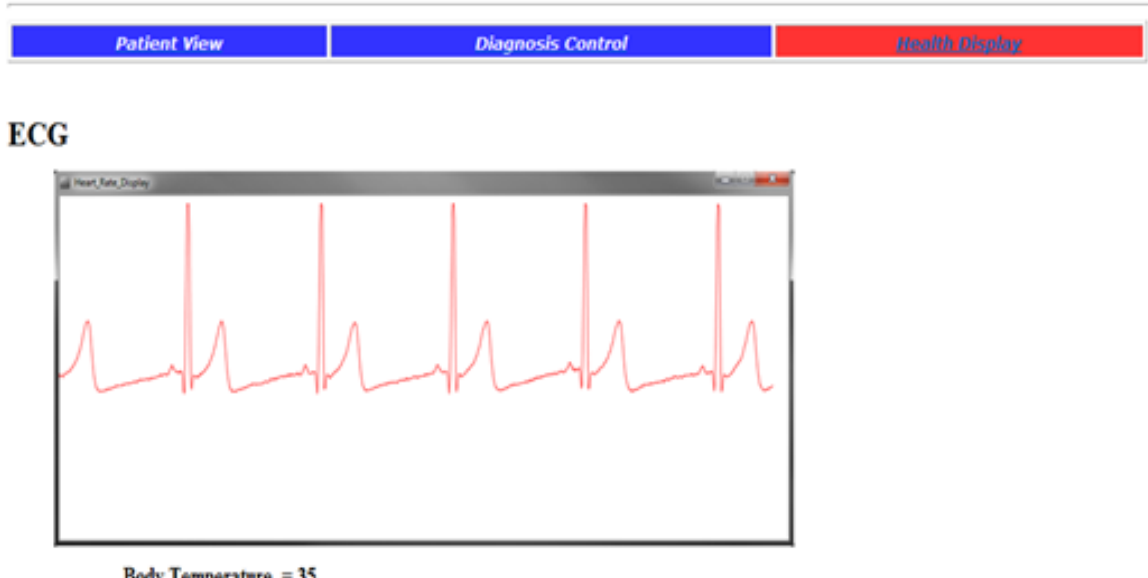

Figure 7. Real time patient health monitoring system

ECG data collected from a patient is shown in fig 8. By comparing the observed values from the normal values heart disease can be diagnosed easily at the early stage. For instance two cycles of ECG waves are chosen and the interval between the two adjacent $\mathrm{R}$ waves (RR interval) is $0.69 \mathrm{~s}$ which denotes there is no irregularity between the two adjacent $\mathrm{R}$ waves. Therefore it is confirmed that the patient's heart rate is healthy and there is no possibility of arrhythmia.

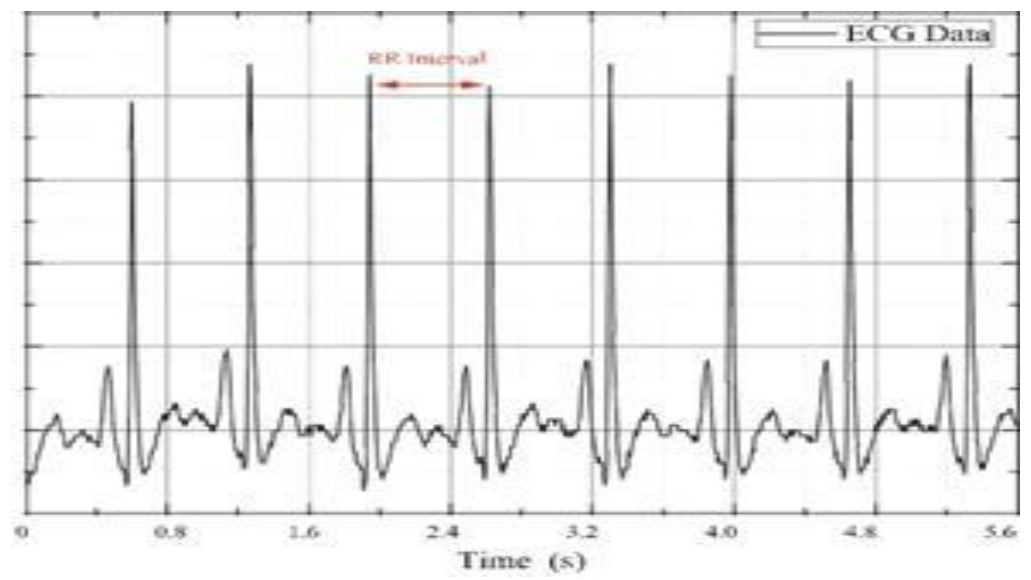

Figure 8. Measured ECG signal 


\section{CONCLUSION}

A continuous remote patient monitoring is important because it is essential for observing health state of patients suffering with acute disease. The health monitoring system must be intelligent enough to deliver real-time analytics, disease prediction and abnormality alert in case of emergency. And this is achieved by internet of thing. This paper proposes a three tier health monitoring system using internet of things which stores and analyse the senor data in the IOT cloud and diagnosis heart disease by comparing the observed ECG data with the normal ECG data. The observed ECG signal shows no irregularities in the RR interval that shows no risk of any type of arrhythmia. Alert message is sent by the system when the health of patients deteriorates. The future work of this study is to implement machine learning technique to diagnose heart disease at the early stage with high accuracy rate.

\section{REFERENCES}

[1] L. Atzori, I. A and G. Morabito, "Internet of Things: a survey," Computer Networks, 2010; vol. 54: pp. 2787-2805,

[2] W. Stallings, Foundations of Modern Networking: SDN, NFV, QoE, IoT, and Cloud. Addison-Wesley Professional, 2015.

[3] H. S. Kim, et al., "A Daily Activity Monitoring System for Internet of Things-Assisted Living in Home Area Networks," International Journal of Electrical and Computer Engineering (IJECE), vol/issue: 6(1), pp. 399-405.

[4] A. Rghioui and A. Oumnad, "Challenges and opportunities of Internet of things in healthcare," International Journal of Electrical and Computer Engineering, 2018; vol. 8, no. 5, pp. 2753-2761.

[5] Lorincz K, Malan DJ, Fulford-Jones TR, Nawoj A, Clavel A, Shnayder V, et al." Sensor networks for emergency response: challenges and opportunities", Pervasive Computing, IEEE, 2014; vol/issue: 3(4), pp.16-23.

[6] Shafiq MZ, Ji L, Liu AX, Pang J, Wang J. "A first look at cellular machine-to-machine traffic: large scale measurement and characterization”, ACM SIG- METRICS Perform Eval Rev 2012; vol 40(1):pp.65-76.

[7] Ishaq I, Carels D, Teklemariam GK, Hoebeke J, Abeele FVD, Poorter ED, et al. "IETF standardization in the field of the Internet of Things (IoT): a survey". J Sens Actuator Networks, 2013; vol/issue:2(2):pp.235-87.

[8] Kumar P, Lee HJ. "Security issues in healthcare applications using wireless medical sensor networks: a survey". Sensors, 2011; vol/issue:12(1),pp.55-91.

[9] Manogaran G, Thota C, Kumar MV. "Meta Cloud Data Storage architecture for Big Data security in cloud computing”. Procedia Computer Science, 2016; vol(3),pp.128-133.

[10] Gubbi J, Buyya R, Marusic S, Palaniswami M. "Internet of Things (IoT): a vision, architectural elements, and future directions". Future Generation Computer Syst, 2016; vol/issue29(7):pp.1645-60.

[11] T. Gao, T. Massey, L. Selavo, D. Crawford, B.-r. Chen, K. Lorincz, V. Shnayder, L. Hauenstein, F. Dabiri, and J. Jeng, "The advanced health and disaster aid network: A light-weight wireless medical system for triage," IEEE Biomedical Circuits and Systems, 2007; vol. 1.

[12] K. Wac, R. Bults, B. Van Beijnum, I. Widya, V. Jones, D. Konstantas, M. Vollenbroek-Hutten, and H. Hermens, "Mobile patient monitoring: the mobihealth system," in IEEE EMBC, 2009.

[13] Varatharajan R, Manogaran G, Priyan M, Bala s VE, Barna C. "Visual analysis of geospatial habitat suitability model based on inverse distance weighting with paired comparison analysis". Multimed Tools Appl, 2017;pp.1-21.

[14] Blum JM, Magill EH. "The design and evaluation of personalised ambient mental health monitors". In: Proceedings of 7th consumer communications and networking conference (CCNC). IEEE, 2010; pp. 1-5.

[15] Deepika.N, Dr.M. Anand, F.Jerald, "Smart and Energy Efficient Medical Sensor Design for Patient Health Care Monitoring”, Journal Of Advanced Research In Dynamical And Control System, 2017; Vol. 9. pp - 1868- 1878. 